\title{
Reflectometria no domínio do tempo em estudos de lixiviação de potássio em colunas de solo não-saturado
}

\author{
Time domain reflectometry for studying potassium leaching in unsaturated soil columns
}

\author{
Anderson Luiz de Souza ${ }^{I}$ Edson Eiji MatsuraII Jarbas Honorio de Miranda ${ }^{\text {III }}$ \\ Marcio Willian Roque ${ }^{\mathrm{IV}}$ Douglas Roberto Bizari ${ }^{\mathrm{IV}}$ Wellington Roque $^{\mathrm{V}}$
}

\section{- NOTA-}

\section{RESUMO}

Este trabalho tem como objetivos projetar e avaliar o potencial de colunas de solo não-saturado equipadas com sondas de TDR (Time domain reflectometry) e acopladas a um sistema automatizado de aquisição de dados no monitoramento da dinâmica da água e de potássio no solo. $O$ ensaio experimental foi conduzido em uma coluna de acrílico de $35 \mathrm{~cm}$ de diâmetro interno e $105 \mathrm{~cm}$ de comprimento preenchida com terra fina seca ao ar. A técnica da TDR possibilitou a obtenção de uma malha espaço-temporal em curtos intervalos de tempo e da umidade e da condutividade elétrica (Ce) nas diferentes profundidades consideradas. Tal resultado pode trazer uma importante contribuição para o entendimento da dinâmica da água e do potássio na terra, auxiliando os trabalhos para proposição e validação de modelos matemáticos que descrevem numericamente esses processos.

Palavras-chave: $T D R$, dinâmica de solutos, coluna de solo não-saturado.

\section{ABSTRACT}

The aim of this research was to evaluate the advantages of using unsaturated soil columns equipped with TDR probes (Time Domain Reflectometry) coupled to an automated system of data acquisition for monitoring water and potassium movement in soils. The research was conducted in acrylic columns of $0.35 \mathrm{~m}$ of internal diameter and $1.05 \mathrm{~m}$ of length filled with air-dried soil. The use of TDR technique allowed obtaining time-space network at short time intervals, indicating levels of soil moisture and electric conductivity (Ce) at different depths. The results may represent an important help for the works on the proposition and validation of mathematical models that describe numerically these processes, contributing to a better understanding of the dynamics of water and potassium in soils.

Key words: TDR, solute movement, unsaturated soil column.

De maneira geral, a avaliação da dinâmica de água e solutos em colunas de solo é feita mediante a extração de amostras de solo, com posteriores quantificações das respectivas umidades gravimétricas e das concentrações dos íons de interesse, mediante o uso de técnicas de extração de solução do solo. Porém, por ser destrutiva, essa técnica não admite a reamostragem de um mesmo ponto de monitoramento, inviabilizando a avaliação da dinâmica de água e solutos ao longo da coluna de maneira contínua, ou seja, ao longo do tempo (SILVA et al., 2005). Nesse sentido, o uso da técnica da reflectometria no domínio do tempo (TDR) em estudos da dinâmica da água e de solutos no solo demonstra um grande potencial, uma

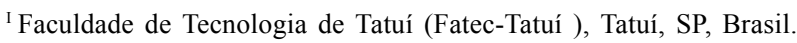

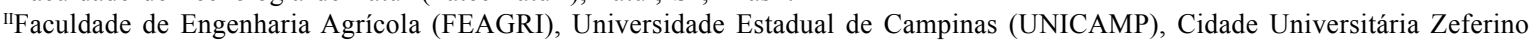
Vaz, Campinas, SP, Brasil. E-mail: matsura@agr.unicamp.br. Autor para correspondência.

IIIDepartamento de Ciências Exatas, Escola Superior de Agricultura Luiz de Queiroz (ESALQ), Universidade de São Paulo (USP), São Paulo, SP, Brasil.

IVPrograma de Pós-graduação na Área de concentração de Água e Solos, FEAGRI, UNICAMP, Campinas, SP, Brasil.

vprograma de Pós-graduação em Engenharia Elétrica, Faculdade de Engenharia Elétrica (FEEC), UNICAMP, Campinas, SP, Brasil. 
vez que, segundo COELHO \& ARRUDA (2001), essa técnica, além de ser bastante precisa, destaca-se justamente por ser um método não-destrutivo, que não utiliza radiação ionizante e pode ser acoplado a multiplexadores, possibilitando a construção de um sistema de aquisição automática de dados em curtos intervalos de tempo (da ordem de alguns segundos).

De um modo geral, a técnica da TDR baseiase no sensível efeito da umidade do solo sobre a velocidade de pulsos de microondas em cabos condutores (guia) envoltos pelo solo. Tal fenômeno deve-se à diferença entre as constantes dielétricas $(\mathrm{Ka})$ da água, do ar e do solo. O equipamento TDR é formado basicamente por um testador de cabos e um sensor com hastes metálicas ligadas entre si por um cabo coaxial, que medem o tempo gasto para um pulso eletromagnético se deslocar do início ao fim da guia de comprimento conhecido (SOUZA et al., 2001). Uma vez que a Ka da matriz do solo afeta a velocidade de deslocamento do pulso eletromagnético, pode-se estabelecer uma correlação entre este tempo de deslocamento e a umidade volumétrica do solo. DALTON et al. (1984), avaliando o uso da TDR para a medição do conteúdo de água no solo, demonstrou que o sinal refletido, além de informações acerca do tempo de trânsito do pulso, contém informações relativas à dissipação da energia eletromagnética que podem ser usadas para avaliar a condutividade elétrica (Ce). Tais características, segundo OR \& JONES (2001), permitem que, de forma indireta, se conheça a condutividade elétrica da solução do solo e a concentração de diferentes nutrientes nessa solução. Dessa forma, a técnica de TDR é uma importante ferramenta nos trabalhos que buscam entendimento da relação água-solo. Dentre as muitas aplicações, segundo SANTANA et al. (2006), destaca-se o uso da TDR no manejo da fertirrigação, tendo em vista que nessa atividade o monitoramento intensivo e contínuo da distribuição de água e de íons aplicados ao solo é condição imprescindível.

Dentre os nutrientes aplicados ao solo na fertirrigação, o potássio destaca-se por ser um macronutriente que na planta associa-se ao desempenho de diferentes funções como: o controle da abertura e do fechamento dos estômatos, a maior translocação de carboidratos produzidos nas folhas para outros órgãos da planta, a maior eficiência enzimática e a melhoria da qualidade comercial da planta (SILVAet al., 2005).

Sendo assim, este trabalho tem como objetivos projetar e avaliar o potencial de colunas de solo não-saturado equipadas com sondas de TDR e acopladas a um sistema automatizado de aquisição de dados a fim de permitir o monitoramento da dinâmica da água e de potássio no solo. Para tanto, utilizou-se uma coluna de acrílico de $35 \mathrm{~cm}$ de diâmetro interno e
$105 \mathrm{~cm}$ de comprimento. Escolheu-se o acrílico, pois, além de ser um material inerte, permite a avaliação visual da acomodação do solo e da frente de molhamento. A coluna foi preenchida com terra fina seca ao ar (TFSA) proveniente de um Latossolo Vermelho Amarelo, fase arenosa, localizado nas coordenadas geográficas de $22^{\circ} 43^{\prime}$ ' 14"de latitude Sul e $47^{\circ} 36^{\prime} 47^{\prime}$ ' de longitude Oeste, no município de Piracicaba-Brasil, cujas características físico-hídricas são apresentadas em MIRANDA\& DUARTE (2002).

Estabelecendo-se uma densidade de $1,5 \mathrm{~kg}$ $\mathrm{dm}^{-3}$, foi calculada a massa de solo seco necessária para o preenchimento do volume total da coluna. Uma vez estabelecida a massa de solo que seria utilizada, buscando-se manter uma densidade homogênea com a ajuda de um funil, a coluna foi preenchida em camadas a partir de sua base. O deslocamento da água e de solutos ao longo da coluna foi monitorado em seis profundidades: $15 \mathrm{~cm}, 30 \mathrm{~cm}, 45 \mathrm{~cm}, 60 \mathrm{~cm}, 75 \mathrm{~cm}$ e $90 \mathrm{~cm}$. Em cada uma dessas profundidades, foram instaladas duas sondas de TDR e um extrator de solução. Foram utilizadas sondas de três hastes de $15 \mathrm{~cm}$ de comprimento, $0,3 \mathrm{~cm}$ de diâmetro e com um espaçamento entre hastes de $2,5 \mathrm{~cm}$, construídas no Laboratório de Hidráulica da Faculdade de Engenharia Agrícola (FEAGRI-UNICAMP). Uma vez instaladas, cada uma das 12 sondas foi acoplada a um multiplexador SDMX50 conectado a um TDR100.

Os extratores de solução utilizados neste trabalho foram construídos utilizando-se cápsulas porosas e mangueiras do tipo cristal de 7,5mm de diâmetro externo e $2 \mathrm{~mm}$ de espessura. A extração de solução foi feita utilizando-se um sistema composto por uma bomba de vácuo com motor elétrico e seis Kitassatos, o que permitiu que um vácuo de $60 \mathrm{kPa}$ fosse aplicado simultaneamente nos diferentes pontos de monitoramento. A concentração de potássio nas soluções amostradas foi determinada mediante o uso de um medidor de íon específico compacto (marca Horiba).

Durante o período de $8 \mathrm{~h} 10 \mathrm{~min}$, por meio de um conjunto de quatro gotejadores distribuídos na superfície da coluna, foi aplicada uma solução de $\mathrm{KNO}_{3}$ numa concentração de potássio de $1500 \mathrm{mg} \mathrm{L}^{-1}$ com uma vazão constante de $3,0 \mathrm{~L} \mathrm{~h}^{-1}$. Utilizando-se um reservatório com uma graduação de $4 \mathrm{~L}$, o controle da vazão aplicada ao longo do experimento foi feito mediante consecutivas avaliações da variação do volume da solução do reservatório em função do tempo.

Uma vez iniciado o experimento, foram feitas, a cada minuto, leituras de $\mathrm{Ka}$ e de $\mathrm{Ce}$ por meio das sondas de TDR. Com o auxilio do software loggernet, tais leituras foram enviadas para um computador portátil, onde foram armazenadas. Além de permitir o armazenamento, o software também permitiu a 
visualização dos dados em tempo real. Para obter uma estimativa da umidade volumétrica $(\theta)$ a partir dos valores de $\mathrm{Ka}$, seguindo a metodologia proposta por TOPP et al.(1980), foi ajustada uma curva de calibração $\left(\mathrm{R}^{2}=0,98\right)$ dada por: $\theta=0,0034 \mathrm{Ka}^{3}-0,2232 \mathrm{Ka}^{2}+5,7554 \mathrm{Ka}-25,519$

Devido à similaridade dos resultados dos pares de sondas instaladas num mesmo ponto de monitoramento, trabalhou-se com as médias desses valores. Na figura 1, são apresentados os valores médios de $\theta$ e Ce nos diferentes pontos de monitoramento ao longo do ensaio experimental. $\mathrm{Na}$ figura $1 \mathrm{~A}$, pode-se verificar que a frente de molhamento atingiu as profundidades de 15, 30, 45, 60, 75 e 90cm com os respectivos tempos de 1, $1 \mathrm{~h}, 2,53 \mathrm{~h}, 3,98 \mathrm{~h}, 5,34 \mathrm{~h}$, $6,66 \mathrm{~h}$ e 7,96h após o início do experimento. Uma vez que a distância entre dois pontos de monitoramento consecutivos foi constante $(15 \mathrm{~cm})$, as variações nos tempos de deslocamento podem indicar uma desuniformidade na compactação do solo ao longo da coluna. Entretanto, o paralelismo das curvas de umidade e o fato de que estas tendem a atingir um mesmo patamar (Figura 1A) sugerem que, para as condições deste experimento, a densidade do solo pode ser considerada homogênea. Na figura 1B, verifica-se que Ce é sensível à frente de molhamento, uma vez que seus valores aumentam com a chegada da mesma. Por outro lado, verifica-se que estas, além de apresentarem um comportamento distinto das curvas de umidade, chegando ao patamar de maneira mais lenta, apresentam comportamentos distintos entre si. Não foi observado paralelismo para as curvas de $\mathrm{Ce}$, sendo o tempo que cada uma delas leva para chegar ao patamar tende a aumentar com a profundidade.

$\mathrm{Na}$ tabela 1, são apresentadas as concentrações de potássio avaliadas a partir das

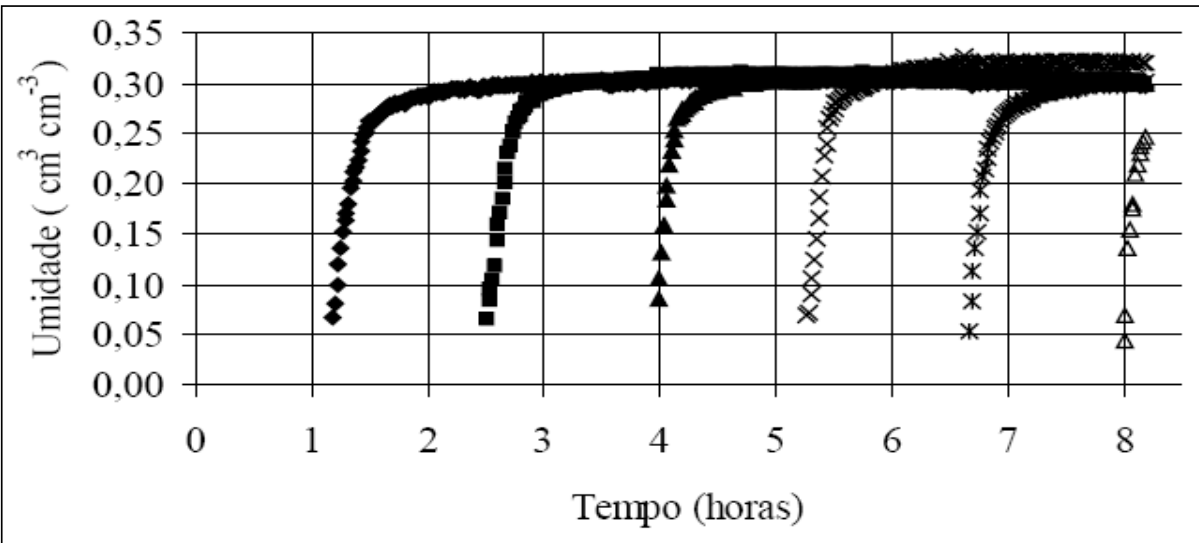

- $15 \mathrm{~cm}=30 \mathrm{~cm} \Delta 45 \mathrm{~cm} \times 60 \mathrm{~cm} * 75 \mathrm{~cm} \Delta 90 \mathrm{~cm}$

(A)

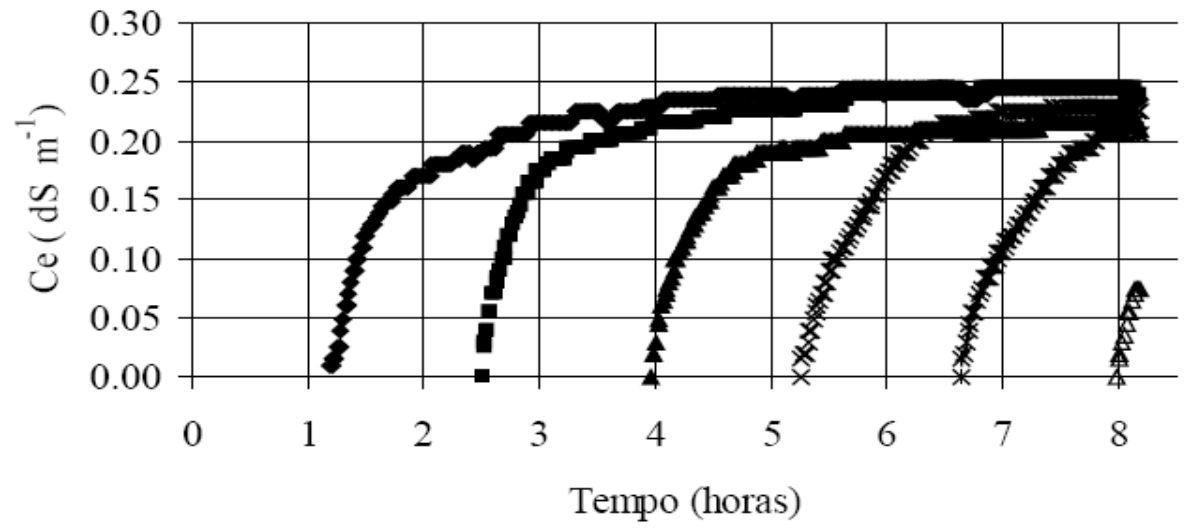

- $15 \mathrm{~cm} \cdot 30 \mathrm{~cm} \Delta 45 \mathrm{~cm} \times 60 \mathrm{~cm} * 75 \mathrm{~cm} \Delta 90 \mathrm{~cm}$

(B)

Figura 1 - Valores médios de è (A) e Ce (B) nas diferentes profundidades ao longo do ensaio experimental.

Ciência Rural, v.39, n.4, jul, 2009. 
Tabela 1 - Umidades e concentrações de potássio nas amostras da solução do solo retiradas pelos extratores de solução nos diferentes pontos de monitoramento.

\begin{tabular}{|c|c|c|c|c|c|c|c|c|c|c|}
\hline \multirow{3}{*}{$\begin{array}{l}\text { Profundidade } \\
\text { (cm) }\end{array}$} & \multicolumn{10}{|c|}{ Tempo (horas ) } \\
\hline & \multicolumn{2}{|c|}{----------2,4---------- } & \multicolumn{2}{|c|}{----------3,5---------- } & \multicolumn{2}{|c|}{----------5,1---------- } & \multicolumn{2}{|c|}{----------6,6---------- } & \multicolumn{2}{|c|}{----------8,1---------- } \\
\hline & $\theta(\%)$ & $\begin{array}{c}\mathrm{C} \\
\left(\mathrm{mg} \mathrm{L}^{-1}\right)\end{array}$ & $\theta(\%)$ & $\begin{array}{c}\mathrm{C} \\
\left(\mathrm{mg} \mathrm{L}^{-1}\right)\end{array}$ & $\theta(\%)$ & $\begin{array}{c}\mathrm{C} \\
\left(\mathrm{mg} \mathrm{L}^{-1}\right)\end{array}$ & $\theta(\%)$ & $\begin{array}{c}\mathrm{C} \\
\left(\mathrm{mg} \mathrm{L}^{-1}\right)\end{array}$ & $\theta(\%)$ & $\begin{array}{c}\mathrm{C} \\
\left(\mathrm{mg} \mathrm{L}^{-1}\right)\end{array}$ \\
\hline 15 & 29,4 & 150 & 30,0 & 990 & 30,4 & 1305 & 30,2 & 1425 & 29,9 & 1425 \\
\hline 30 & 2,7 & - & 30,1 & 45 & 30,7 & 15 & 30,5 & 720 & 30,0 & 1155 \\
\hline 45 & 5,2 & - & 4,9 & - & 30,3 & 45 & 30,3 & 30 & 30,1 & 30 \\
\hline 60 & 5,3 & - & 6,1 & - & 5,3 & - & 32,2 & 22,5 & 32,1 & 15 \\
\hline 75 & 3,8 & - & 3,6 & - & 3,4 & - & 2,1 & - & 30,0 & 60 \\
\hline 90 & 2,1 & - & 2,0 & - & 2,1 & - & 2,1 & - & 23,5 & - \\
\hline
\end{tabular}

amostras da solução do solo retiradas pelos extratores de solução e as respectivas umidades obtidas por meio da técnica da TDR. Verifica-se que, para uma mesma profundidade, a concentração de potássio na solução extraída do solo tende a aumentar com o tempo. Além disso, considerando diferentes profundidades, embora os gradientes de umidade tenham diminuído consideravelmente, as concentrações de potássio foram maiores nas camadas superiores. O comportamento das curvas apresentadas tanto na figura $1 \mathrm{~B}$ e os resultados da tabela 1 justificam-se à medida em que os mecanismos envolvidos no transporte do potássio baseiam-se, sobretudo, na rápida troca com outros cátions adsorvidos à matriz do solo. Num primeiro momento, à medida em que a solução deslocase através da coluna, parte do potássio em solução fica adsorvida à matriz do solo e, conseqüentemente, há um decréscimo na concentração desse íon à medida em que a solução desloca-se em profundidade. Com o decorrer do tempo, a partir das camadas superiores, começa a haver uma redução da capacidade do tampão do potássio e assim o transporte desse íon para as camadas mais profundas torna-se mais efetivo. Pela consistência dos dados obtidos por meio das sondas de TDR, verifica-se que estes foram bastante sensíveis, tanto ao deslocamento de água, quanto ao deslocamento do potássio ao longo da coluna de solo. Isso demonstra que o uso de colunas de acrílico equipadas com sondas de TDR trouxe novos avanços no monitoramento e no entendimento dos processos envolvidos na dinâmica da água e do potássio em condições de solo não-saturado.

\section{AGRADECIMENTOS}

À Fundação de Amparo à Pesquisa do Estado de São Paulo (FAPESP), pelo financiamento do projeto, e à Coordenação de Aperfeiçoamento de Pessoal de Nível Suoerior (CAPES) e ao Conselho Nacional de Desenvolvimento Científico e Tecnológico (CNPq), pela concessão da bolsa.

\section{REFERÊNCIAS}

COELHO, E.F.; ARRUDA, F.B. Aplicações da técnica de TDR: manejo da água no solo. In: MATSURA, E.E. et al. Aplicações da técnica de TDR na agricultura. Campinas: Feagri/ Unicamp, 2001. Inserir as páginas inicial e final do capítulo ou seção.

DALTON, F.N.et al. Time domain reflectometry: simultaneous measurement of soil water content and electrical conductivity with a single probe. Science, n.224, p.989-900, 1984. Disponível em: <http://www.sciencemag.org/cgi/content/ abstract/224/4652/989>. Doi: 10.1126/science.224.4652.989.

MIRANDA, J.H.; DUARTE, S.N. Modelo para simulação da dinâmica de nitrato em colunas verticais de solo não saturado. Revista Brasileira de Engenharia Agrícola e Ambiental, v.6, n.2, p.235-241, 2002.

OR, D.; JONES, S.B. Extending TDR measurements range in saline soils using frequency-domain methods. In: MATSURA, E.E. et al. Aplicações da técnica de TDR na agricultura. Campinas, Feagri/Unicamp, 2001. P.

SANTANA, G. S.et al. Relação entre potássio na solução do solo, umidade e condutividade elétrica aparente do solo. Revista Brasileira de Engenharia Agrícola e Ambiental, v.11, n.2, p.142-151, 2006. Disponível em: < http://www.scielo.br/ scie lo.php? script=sci_arttext\&pid=S 1415 $43662007000200003 \& \operatorname{lng}=$ en $\&$ nrm $=$ iso\&tlng $=\mathrm{pt}>$. Doi: $10.1590 /$ S $1415-43662007000200003$.

SILVA, T.S.M. et al. Teor de potássio na solução do solo com uso da técnica de reflectometria no domínio do tempo. Irriga, v.10, n.4, p.393-402, 2005.

SOUZA, C.F. et al. Experiência do laboratório de hidráulica, irrigação e drenagem na Faculdade de Engenharia Agrícola/ UNICAMP no uso da técnica de TDR. In: MATSURA, E.E. et al. Aplicações da técnica de TDR na agricultura. Campinas, Feagri/Unicamp, 2001. 147-176p.

TOPP, G.C. et al. Electromagnetic determination of soil water content: measurements in coaxial transmission lines. Water Resources Research, v.16, p.574-582, 1980.

Ciência Rural, v.39, n.4, jul, 2009. 